\title{
Soziale Arbeit, nach acht
}

Arbeitsfeld Nightlife

Alexander Bücheli ${ }^{1}$

\section{Einleitung}

Die Soziale Arbeit blickt im Bereich Nachtleben in Europa auf eine über dreissigjährige Geschichte zurück. Die ersten Präventionsangebote wurden Mitte der 1980er-Jahre in Holland ins Leben gerufen. Anlass dafür waren eine sich schnell ausbreitende Rave-Kultur, die damit einhergehende Verbreitung des XTC-Konsums ${ }^{2}$ und die Erfahrung, dass die damaligen, oft auf Abschreckung abzielenden Präventionsangebote in der Partyszene nur auf wenig Akzeptanz stiessen. Bei den ersten Nightlife-Präventionsangeboten ging es deshalb darum, innovative Projekte mit aufsuchendem und akzeptierendem Ansatz aufzubauen (vgl. Bücheli et al. 2017: 43). Die ersten Präventionsangebote wurden dabei oft von PartygängerInnen selbst, die aufgrund eigener Erfahrungen mit den Risiken des Konsums psychoaktiver Substanzen konfrontiert waren, ins Leben gerufen. Dank ihrer Rolle als erfahrene Party-Peers und ihrer aktiven Teilnahme in der Lebenswelt Nachtleben konnte die bis dato schwierig zu erreichende Zielgruppe erreicht werden. Schnell wurde dieser Peer-to-Peer-Ansatz von der Sozialen Arbeit, insbesondere aus dem Fachbereich der offenen Jugendarbeit, unterstützt und adaptiert. Mitte der 1990er-Jahre entstanden auch in den Schweizer Städten Nightlife-Präventionsangebote, die sich direkt an Freizeitdrogenkonsumierende richteten, die an Partys oder Festivals psychoaktive Substanzen einnahmen. Die Angebote verfolgten das

\footnotetext{
12001 bis 2015 zuständig für das Stadtzürcher Nightlife-Präventionsangebot der Jugendberatung Streetwork von saferparty.ch. Mitbegründer des Vereins Safer Clubbing Schweiz und der Bar \& Club Kommission Zürich. Seit 2015 freischaffender Berater für die Netzwerke Safer Dance Swiss, Safer Nightlife Schweiz und Safer Clubbing Schweiz sowie Lobbyist für die Bar \& Club Kommission Zürich, die Schweizer Bar- und Clubkommission und PromoterSuisse. ${ }^{2}$ XTC ist das Kürzel für eine in den 1990er-Jahren aufgekommene Partydroge, deren Wirkstoff MDMA ist. Heute auch bekannt als MDMA, MDI etc.
} 
Ziel, junge Menschen so aufzuklären, dass sie die Lebensphase des Partyfeierns körperlich und psychisch möglichst unbeschadet überstanden. In dieser Zeit entstanden die bis heute existierenden Drogeninformationszentren in Bern und Zürich. Die Angebote sind weiterhin niederschwellig, kostenlos und anonym nutzbar (vgl. Bücheli et al. 2017: 43). Heute wird der Begriff Nightlife-Prävention weiter gefasst und umfasst sowohl schadensmindernde als auch präventive Aspekte und die Zielgruppe wurde auf Freizeitdrogenkonsumierende die nicht in Clubs oder Festivals konsumieren ausgeweitet. In den letzten Jahren rückten verstärkt Ansätze der Sozialen Arbeit in den Fokus, die sich mit den strukturellen Gegebenheiten des Nachtlebens auseinandersetzen. Beispielsweise setzt sich der Verein Safer Clubbing Schweiz gemeinsam mit den Clubs und seinen Mitgliedern für freiwillige strukturelle Anpassungen der Rahmenbedingungen (z. B. Ruhezonen) ein. Relativ neu ist, dass sich auch die Fachbereiche Gemeinwesenarbeit und Stadtentwicklung vermehrt mit dem nächtlichen Vergnügen und dessen Auswirkungen auf die räumliche Umgebung auseinandersetzen (vgl. Bücheli 2015: 25).

\section{Beschreibung des Arbeitsfeldes Nachtleben}

Es gibt keine Definition für den Begriff Nachtleben. Meist werden damit Aktivitäten bezeichnet, abends und in der Nacht auszugehen und sich zu amüsieren. Diese Freizeitaktivität wird umgangssprachlich «Weggehen», «Partymachen», «Clubben» oder «Ausgehen» genannt. Ende der 1990erJahre wurden in vielen Deutschschweizer Städten die Gastgewerbegesetze liberalisiert, ${ }^{3}$ was zu einem Boom von neuen Clubs und Bars führte. In den Sommermonaten wird das Nachtleben heutzutage durch eine Vielzahl von Musikfestivals ergänzt. Dabei kann es sich um reine Tanzveranstaltungen, wie z. B. die Street Parade, oder um Livemusik-Festivals mit Dance-Acts (z. B. DJs) handeln. Die zunehmende Reglementierung, z. B. das Rauchverbot, förderte diese Entwicklung und führte auch zu einem

\footnotetext{
${ }^{3}$ Neben der Herabsetzung der Hürden für das Eröffnen eines Gastgewerbebetriebes, z. B. die Abschaffung der Wirteprüfung, wurden vor allem die Öffnungszeiten liberalisiert. In der Stadt Zürich können Betriebe seit der Liberalisierung des Gastgewerbegesetzes z. B. am Wochenende rund um die Uhr geöffnet haben.
} 
Revival von privaten und illegalen Anlässen, wie man sie vor der Liberalisierung gekannt hatte.

Eine Nightlife-Area ist ein geographisch abgegrenzter öffentlicher Raum, meist Teil eines Quartiers, mit einer hohen Bar- und Clubdichte. Typische nächtliche Stakeholder sind Bar- und ClubbesitzerInnen und Festival-OrganisatorInnen, aber auch einzelne BehördenvertreterInnen, SozialarbeiterInnen, AnwohnerInnen und die Polizei stehen mit dem Nachtleben in enger Verbindung. Seit einigen Jahren setzt man sich mit dem Nachtleben auch als spezifischem Sozialraum auseinander. Ein NightlifeSozialraum besteht dabei aus Locations, deren Infrastruktur und Stakholder sowie der unmittelbaren räumlichen Umgebung und wird von MusikerInnen, GrafikerInnen, LichtkünstlerInnen und unterschiedlichsten PartygängerInnen frequentiert. Ein Nightlife-Sozialraum ist räumlich nicht klar abgegrenzt, das heisst, er folgt keinen Stadteilgrenzen und steht im permanenten Austausch mit anderen Sozialräumen, z. B. mit denjenigen eines Quartiers, in welchem sich Angebote des Nachtlebens befinden (vgl. Bücheli 2010: 16).

Die Motivation zu feiern basiert auf der möglichen Befriedigung unterschiedlichster menschlicher Bedürfnisse, sei es das Bedürfnis nach sozialen Kontakten, Abwechslung oder Entspannung. Ausgehen nimmt innerhalb der heutigen Gesellschaft eine wichtige Funktion ein, wenn es darum geht, vom Alltag abzuschalten (vgl. Kosnick 2011). Als PartygängerInnen bezeichnet man Personen, die regelmässig Clubs, Partys oder Festivals jeglicher Art besuchen. Da die meisten Veranstaltungen ein Mindestalter für den Eintritt fordern - Clubs in der Regel 18 Jahre - machen junge Erwachsene zwischen 18 und 30 Jahren den Hauptanteil aus.

\section{Herausforderungen für die Soziale Arbeit in der Nacht}

Der Fokus der Sozialen Arbeit im Nachtleben liegt seit den 1990er-Jahren auf der Gesundheit der Partygäste. Im Mittelpunkt stehen die personenund umfeldbezogenen Risiken, die häufig in Verbindung mit dem Konsum legaler oder illegaler psychoaktiver Substanzen stehen. Ziel ist es, risikoarmes Verhalten zu fördern. Dazu gehört auch eine Zusammenarbeit 
mit den Nachtkulturunternehmen ${ }^{4}$, um diese für die Anliegen der Prävention und Schadensminderung zu sensibilisieren. Der Öffentlichkeitsarbeit kommt eine wichtige Funktion zu, da Konsum und Konsumkontext von illegalen Substanzen schnell zu sozialen Spannungen führen können. Dadurch steht nicht nur die Soziale Arbeit im Nachtleben unter besonderer öffentlicher Aufmerksamkeit, das Spannungsfeld führt auch dazu, dass die Realität des Drogenkonsums in der Nacht durch die Nachtkulturunternehmen aus Angst vor Administrativmassnahmen ${ }^{5}$ oft verleugnet wird. $\mathrm{Zu}$ den Herausforderungen zählt aber auch das nächtliche Gemeinwohl, also die Koexistenz zwischen den PartygängerInnen und den Anwohnenden. Nachbarschaftskonflikte, z. B. bei Lärmproblemen, sind ein Beispiel dafür, wo Soziale Arbeit heute als Konfliktvermittlerin gefragt ist. Weitere Betätigungsfelder der Sozialen Arbeit sind z. B. die soziokulturelle Animation und die niederschwellige Jugendarbeit, wo das Durchführen von Partys zum Angebot gehört. Auf diese Tätigkeitsfelder der Sozialen Arbeit mit Berührungen zum Nachtleben wird in diesem Artikel nicht weiter eingegangen.

\subsection{Personen- und umfeldbezogene Risiken}

Prinzipiell kann zwischen personenbezogenen, umfeldbezogenen und substanzbezogenen Risiken unterschieden werden (vgl. Bachmann \& Bücheli 2011). Umfeldbezogene Risiken sind z. B. hohe Musiklautstärke, (zu) hohe BesucherInnenzahlen, mangelnde Raumlüftung oder fehlender Trinkwasserzugang. Aufgrund der hohen Regulierungsdichte ${ }^{6}$ und strengen Bewilligungspraxis spielen solche Risiken in der Schweiz eine eher untergeordnete Rolle. Mit personenbezogenen Risiken sind primär Verhaltensweisen der PartygängerInnen gemeint (vgl. ebd.). Von besonderer Bedeutung sind hier der problematische Konsum von psychoaktiven Sub-

\footnotetext{
${ }^{4}$ Der Begriff Nachtkulturunternehmen bezieht sich auf Kulturunternehmen, die primär in der Nacht aktiv sind und umfassen neben Bars, Clubs und Eventlocations auch Festivals.

${ }^{5}$ Administrativmassnahmen in Bezug auf Drogenkonsum in Bars oder Clubs können bis zum Entzug des Patentes führen.

${ }^{6}$ Beispielsweise die nationale Schall- und Laserverordnung, die kantonalen Feuerschutzauflagen etc.
} 
stanzen sowie - häufig damit verbunden - riskante Sexualkontakte, gewalttätige Konfliktbewältigung und Risikoverhalten im Strassenverkehr (vgl. Maier et al. 2013: 23).

Die Resultate verschiedenster Studien weisen auf weitaus höhere Prävalenzen des Konsums von legalen und illegalen Substanzen bei PartygängerInnen im Vergleich zur Gesamtpopulation hin (vgl. Maier et al. 2013: 22). Dies hat damit zu tun, dass am Nachtleben tendenziell jüngere Menschen teilnehmen; eine Gruppe, die im Vergleich zur übrigen Bevölkerung überhaupt die höchsten Konsumprävalenzen aufweist. Das Nachtleben bietet sich in seiner Kombination von Musik und Tanz für Rauscherfahrungen an. Dabei werden die Effekte des Konsums von jungen PartygängerInnen oft als positiv bewertet. Die konsumierten Substanzen können dem Abschalten und dem Entspannen dienen oder dazu beitragen, dass Musik intensiver wahrgenommen und in der Erinnerung verfestigt wird (vgl. Müller \& Schumann 2011: 295). Eine Rangliste der am häufigsten konsumierten Substanzen zeigt, dass Alkohol die beliebteste Partydroge ist, gefolgt von Tabak und Cannabis. Erst mit einigem Abstand werden XTC (MDMA), Kokain und Amphetamin (Speed) konsumiert (vgl. Wollschläger \& Schori 2019: 6). Neben dem Konsum einzelner Substanzen werden im Rahmen einer Partynacht oder eines Festivalbesuches häufig unterschiedliche Substanzen miteinander kombiniert (vgl. ebd.: 13. Ein solcher Mischkonsum stellt aufgrund möglicher, nur schwer voraussehbarer Wechselwirkungen eine besonders problematische Konsumform dar. Obwohl es sich beim Freizeitdrogenkonsum im Nachtleben meist um einen unregelmässigen und nur selten abhängigen Konsum handelt, gibt es klare Anzeichen für problematische Konsummuster und damit verbundene psychische Probleme. Oft haben diese Konsumierenden noch keine professionelle Hilfe in Anspruch genommen oder suchen diese erst zu einem späten Zeitpunkt der problematischen Konsumentwicklung auf (vgl. Maier et al 2014: 13).

Zur Gefahr von substanzbezogenen Nebenwirkungen oder einer substanzgebundenen Abhängigkeit und den Risiken des Mischkonsums kommt im Falle von illegalen Substanzen noch dazu, dass objektive Informationen zu den tatsächlich enthaltenen Inhaltsstoffen und deren Gehalt 
fehlen. Dadurch besteht immer ein gewisses Risiko, dass eine ungewollte - weil nicht deklarierte - Substanz oder eine zu hohe Dosis eingenommen wird.

In den Fokus der medialen Berichterstattung ist in den letzten Jahren ausserdem der Zusammenhang von Nightlife und gewalttätigem Verhalten gerückt. Auch wenn das Nachtleben, betrachtet man die grosse Masse der NachtschwärmerInnen, als friedlich bezeichnet werden kann, kommt es im Umfeld von Bars, Clubs und Festivals immer wieder zu Gewaltvorfällen und sexuellen Übergriffen. Begünstigt werden diese zumindest teilweise durch den Konsum von Alkohol und anderen psychoaktiven Substanzen. Insbesondere Alkohol kann wegen seiner enthemmenden und aggressionssteigernden Wirkung als Katalysator, wenn auch nicht als Ursache von Gewalt bezeichnet werden. TäterInnen fühlen sich in der Nacht anonymer und somit geschützter als am Tag.

\section{Prävention und Schadensminderung im Nachtleben}

Unter Nightlife-Präventionsangeboten werden unterschiedliche Angebote zusammengefasst. Diese haben einen Fokus auf den personenbezogenen Risiken und beinhalten sowohl schadensmindernde als auch präventive Aspekte. Die Zielgruppe wurde im Laufe der Zeit von den Partydrogenkonsumierenden auf die übrigen Freizeitdrogenkonsumierende ausgeweitet. Die Angebote sind - wie bereits die Schweizer Pionierprojekte niederschwellig, das heisst kostenlos und anonym nutzbar.

\subsection{Schweizer Pionierprojekte}

Die ersten Interventionen erfolgten durch szenennahe Organisationen, welche nach dem Peer-to-Peer-Ansatz durch PartygängerInnen selbst ins Leben gerufen wurden. Sie hatten das Ziel, direkt vor Ort neutrale Informationen über Wirkungen und Nebenwirkungen von Partydrogen sowie 
Safer-Use-Regeln ${ }^{7}$ zu vermitteln. ${ }^{8}$ Die Stadt Zürich baute 1995 unter Federführung des damaligen Instituts für Sozial- und Präventivmedizin der Universität Zürich (ISPMZ) ${ }^{9}$ das Projekt XTC-Info auf. Nachdem 1996 zwei unabhängig voneinander erstellte Rechtsgutachten feststellten, dass Drug Checking in der Schweiz als Massnahme der Schadensminderung gegen kein geltendes Recht verstösst, begann der Verein Eve\&Rave Schweiz im Rahmen eines einjährigen Pilotprojektes an Raves Substanzen zur Analyse entgegenzunehmen und die Resultate auf der vereinseigenen Website zu veröffentlichen. 1998 entstand in Bern das erste mobile Drug-CheckingAngebot Pilot E. Dabei handelte es sich um eine Kooperation zwischen der heutigen Stiftung Contact und der Gesundheits- und Fürsorgedirektion des Kantons Bern. 2001 wurde dieser Ansatz in der Stadt Zürich in das Partydrogenpräventionsangebot der Jugendberatung Streetwork integriert und mit der Warnungs- und Substanzinformationsseite saferparty.ch ergänzt (vgl. Bücheli 2014). Im Bereich der Strassensicherheit wurde 2002 von der Fondation vaudoise contre l'alcoolisme (FVA) das Projekt «be my angel tonight» entwickelt, welches seit 2006 durch die Fachstelle «Alkohol am Steuer nie» (ASN) auch in der Deutschschweiz angeboten wird.

Auf struktureller Ebene entwickelte die Plattform Nightlife des Groupement Romand d'Etudes des Addictions (GREA) eine Veranstaltungs-Charta. 2004 rief die Jugendberatung Streetwork in enger Zusammenarbeit mit den Zürcher Clubs und aufbauend auf dem seit 2002 durchgeführten Round Table Nightlife das Gütesiegel Safer Clubbing ins Leben. Streetwork war es auch, die 2006 die erste stationäre Drug-Checking-Möglichkeit im Rahmen des Drogeninformationszentrums DIZ schuf und einen integrativen Partydrogenpräventionsansatz unter dem Brand saferparty.ch zu entwickeln begann. Seit 2006 besteht eine regelmässige Zusammenarbeit, sowohl innerhalb der Plattformen Nightlife der GREA und der Fachgruppe Nightlife des Fachverbands Sucht, als auch zwischen den bei-

\footnotetext{
7 Safer-Use-Regeln sind Handlungsanweisungen, welche darauf abzielen, einen potentiellen Schaden zu verhindern oder zumindest das Schadenspotential zu minimieren.

8 Als immer noch aktive Organisation ist hier der 1996 gegründete Verein Eve\&Rave Schweiz zu nennen.

${ }^{9}$ Heute Institut für Epidemiologie, Biostatistik und Prävention (EPBI).
} 
den Netzwerken auf nationaler Ebene, z. B. im Rahmen spezifischer Arbeitsgruppen. 2012 wurde unter der Führung von Infodrog das nationale Kompetenznetzwerk Safer Nightlife Schweiz (SNS) gegründet mit dem Ziel, den interdisziplinären Wissenstransfer zu fördern. (vgl. Bücheli 2014).

\subsection{Die Rolle der Sozialen Arbeit}

Auch wenn es sich beim Konsum psychoaktvier Substanzen eigentlich um ein Gesundheitsthema handelt, nimmt die Soziale Arbeit in Bezug auf die Prävention und Schadensminderung im Nachtleben eine wichtige Rolle ein. Zu Beginn, in den 1990er-Jahren, unterstützte die Soziale Arbeit die anfänglich oft informellen, auf freiwilligem Engagement basierenden Peer-to-Peer-Ansätze. Der szenennahe Ansatz wurde zunehmend adaptiert und erste professionelle Nightlife-Präventionsangebote als Angebote der Sozialen Arbeit entwickelt. Dank der nationalen Zusammenarbeit innerhalb der Netzwerke und zwischen den einzelnen Nightlife-Präventionsangeboten - nicht nur in Bezug auf gemeinsam genutzte Tools, sondern auch auf der Ebene von Weiterbildungen - wurde der Bereich zunehmend professionalisiert. Heute sind die Tätigkeiten der «Sozialen Arbeit nach acht» so vielfältig wie der Sozialraum Nachtleben. Es kann sich dabei um eine Tätigkeit mit direktem Kontakt zur Zielgruppe handeln, beispielsweise als Berater im Rahmen eines Nightlife-Präventionsangebotes. Anderseits sind auch koordinierende oder beratende Funktionen gefragt, bei welchen es z. B. um das konkrete Management (z. B. SIP) und/oder die Entwicklung des nächtlichen Sozialraumes (z. B. Stadtentwicklung, Quartierkoordination) geht. Da die meisten Angebote im sozialen Bereich verankert sind und durch städtische oder kantonale Sozialdepartemente finanziert werden, ist eine Nähe zur Sozialen Arbeit schon strukturell gegeben.

Grundlagen für die Prävention im Nachtleben sind eine akzeptanzorientierte Haltung ${ }^{10}$ und der aufsuchende Ansatz. Die Angebote der Prävention und Schadensminderung arbeiten dabei vernetzt, orientieren sich

\footnotetext{
${ }^{10}$ Akzeptanzorientiert heisst, dem Konsum illegaler Drogen neutral und ohne zu moralisieren begegnen. Das Ziel ist nicht die Abstinenz, sondern die bestmögliche Gesundheit inner-
} 
an Fakten und stützen sich in der Schweiz auf die Resultate einer kontinuierlichen Befragung der erreichten Zielgruppe und auf die Drug-Checking-Analyseresultate ab. Am individuellen Risikoverhalten orientierte, selektiv vermittelte Informationen sollen zu einer möglichst risikoarmen Haltung beim Konsum psychoaktiver Substanzen führen (vgl. Bücheli 2017). Zur Optimierung des Angebots wurden in den letzten Jahren zunehmend Kurzberatungs-, Früherkennungs- und Frühinterventionsansätze ${ }^{11}$ für die Anwendung im Nachtleben angepasst (vgl. Bachmann et al. 2013: S. 43). Für Risikosituationen wie das Lenken eines Fahrzeugs unter Alkoholeinfluss wurden spezifische Angebote wie «be my angel tonight», entwickelt.

Um die Umfeldrisiken anzugehen und strukturelle Verbesserungen im Sozialraum Nachtleben zu erzielen, wird verstärkt auf die Zusammenarbeit mit den Nightlife-Stakeholdern wie Bar- und Club-BetreiberInnen gesetzt. Zur Anwendung kommen dabei Ansätze der Gemeinwesenentwicklung. Bei nächtlichen Konflikten und Fragen der friedlichen Koexistenz zwischen PartygängerInnen und Anwohnenden spielt die Mediation eine wichtige Rolle. In den folgenden Abschnitten werden verschiedene Ansätze der Sozialen Arbeit im Nachtleben diskutiert.

\subsubsection{Grundlagenarbeit}

Als Grundlagenarbeit versteht man bei der Sozialen Arbeit im Nachtleben vor allem das Sammeln und Aufbereiten von relevantem Wissen. Sozialarbeitende sind typischerweise für das Sammeln und Bereitstellen von Informationen sowie für die Pflege eines interdisziplinären Informationsnetzwerkes ${ }^{12}$ zuständig. Ziel der Grundlagenarbeit ist, Fakten und Safer-

\footnotetext{
halb eines selbstgewählten Lebensentwurfs. Dazu gehört das Vermitteln von Safer-Use-Botschaften und von Fakten. Akzeptanzorientiertheit heisst dabei nicht, dass jeder Lebensentwurf als gegeben akzeptiert werden muss, Beratungsgespräche bieten auch weiterhin die Möglichkeit, gewählte Entwürfe kritisch zu hinterfragen, Alternativen aufzuzeigen.

${ }^{11}$ Dabei geht es nicht um eine Früherkennung im klassischen Sinne, da die AngebotsnutzerInnen ja meist schon mit dem Konsum psychoaktiver Substanzen begonnen haben. Vielmehr geht es um die Früherkennung der Entwicklung besonders problematischer Konsumformen, um den Beginn eines abhängigen Konsums zu verhindern.

${ }_{12}$ Diese Netzwerke umfassen Fachleute aus den Bereichen Forensik, Therapie und Psychologie sowie aus der Grundlagenforschung, wenn es um die Wirkung und Risiken von psychoaktiven Substanzen geht.
} 
Use-Anleitungen zu schaffen, an welchen sich Konsumierende orientieren können. Ideal ist, wenn zur Faktengewinnung auf Daten von Drug-Checking-Angeboten zurückgegriffen werden kann. Ein oft unterschätzter Wissensträger sind die PartygängerInnen, weshalb ein Beratungsgespräch immer auch zu einem Wissensgewinn führen kann. Wissen, welches vor der Veröffentlichung anonymisiert, gefiltert und interpretiert werden muss. Die Beratung vor Ort hat somit auch eine gewisse Monitoring-Funktion. Für die Interpretation und Gewichtung der gesammelten Informationen ist meist ein multidisziplinäres Netzwerk verantwortlich. Eine weitere Form der Grundlagenarbeit ist die Entwicklung gemeinsamer Tools, beispielsweise die Informationsbroschüre «Drugs - Just Say Know» ${ }^{13}$, der bei den Drug Checkings national verwendete Fragebogen, die Entwicklung von Beratungsstandards (vgl. Infodrog 2018) und das Anbieten von spezifischen Weiterbildungen für die im Bereich der Nightlifeprävention tätigen Peers und Fachpersonen.

\subsubsection{Nightlife-Präventionsangebote}

Nightlife-Präventionsangebote setzen inhaltlich primär auf Risikosensibilisierung und Safer-Use-Informationen. Ziel ist Schadensminderung, dazu gehört das Vermitteln eines risikoarmen Umgangs mit psychoaktiven Substanzen. Die Mitglieder der Zielgruppe werden dabei als Individuen betrachtet, die in den meisten Fällen über die nötigen Ressourcen verfügen, um Wissen in Verhaltensänderungen umzuwandeln. Basis für die Intervention vor Ort ist meist ein durch Fachpersonal (SozialarbeiterInnen und/oder Peers) betreuter Infostand. Die Vermittlung selektiver Informationen findet im Rahmen von Kurzberatungen statt. Hier steht das persönliche Risikoverhalten im Mittelpunkt des Gesprächs. Als für die Zielgruppe besonders attraktiv haben sich Nightlife-Präventionsangebote mit integriertem Drug Checking herausgestellt (vgl. Schori 2019: 10).

\footnotetext{
${ }^{13}$ DRUGS - JUST SAY KNOW informiert über psychoaktive Substanzen, ihre Wirkungen, Nebenwirkungen und Risiken und weist auf Möglichkeiten des Safer Use hin. Das Infoset umfasst allgemeine Informationen zum Drogenkonsum und Informationen zu 30 bewusstseinsverändernden Substanzen sowie zum verbreiteten Mischkonsum. www.know-drugs.ch
} 


\subsubsection{Drug Checking}

Drug-Checking-Angebote beinhalten neben einer Substanzanalyse immer auch ein persönliches Beratungsangebot zu Substanzkonsum und Risikoverhalten (vgl. Infodrog 2017: 1). Das Ziel ist es, unerwünschte Nebenwirkungen des Konsums aufgrund hoher Dosen, unerwarteter Substanzen oder Streckmittel zu verhindern. Die Beratung ist Teil des Nachtleben-Präventionsangebots (mobiles Drug Checking) oder wird in den Drogeninformationszentren (ambulantes Drug Checking) angeboten. Beim mobilen Drug Checking handelt es sich um ein Nightlife-Präventionsangebot mit integriertem Labor, welches direkt im Club oder am Festival aufgestellt wird. Die durch Fachpersonen, z. B. Chemiker, durchgeführte Substanzanalyse findet vor Ort statt und dauert etwa 20 Minuten. Während dieser Zeit führt einE MitarbeiterIn der Prävention mit der Person, die eine Substanz abgegeben hat, ein obligatorisches Kurzberatungsgespräch durch und füllt mit deren Einverständnis einen standardisierten anonymen Fragebogen aus. Nach der Analyse wird die Person, welche die Substanz abgegeben hat, direkt über die Resultate (Streckmittel, Reinheit, Wirk- und Risikopotenzial, Safer Use) informiert. In Zürich (seit 2006), Bern (seit 2014), Basel (seit 2018), Genf (seit 2019) existieren ambulante Drug-Checking-Angebote, wo an einem festen Standort Substanzen zum Testen abgegeben werden können. ${ }^{14}$ Die Resultate werden nach einigen Tagen per Mail oder Telefon mitgeteilt. Neben der Zugänglichkeit für eine breitere Zielgruppe bieten diese Angebote auch weiterführende Kurzberatungsgespräche und/oder die Triage in eine spezialisierte Institution an.

\subsubsection{Virtuelle Informationskanäle}

Für die selektive Informationsvermittlung werden aber nicht nur Nightlife-Präventionsangebote vor Ort genutzt, sondern zunehmend auch virtuelle Kommunikationskanäle und soziale Medien. Anfänglich handelte es sich vor allem um Websites, die Informationen zu Wirkung, Nebenwirkung und Langzeitfolgen von Partydrogen enthielten. Heute werden vermehrt auch Apps und Social-Media-Kanäle wie Facebook und Twitter zur Informationsvermittlung genutzt. Das virtuelle Angebot umfasst nicht mehr lediglich Substanzinformationen und Warnungen, es beinhaltet

\footnotetext{
${ }^{14}$ In Luzern ist ein solches Angebot für 2020 geplant.
} 
auch Links zu weiterführenden Beratungsangeboten, Konsumtagebücher oder Onlineberatungsangebote. $\mathrm{Zu}$ den beiden bekanntesten Websites in der Schweiz gehören www.saferparty.ch und www.eve-rave.ch.

\begin{tabular}{|c|c|c|c|}
\hline Angebot & Beschreibung & Ziele & Referenzen \\
\hline $\begin{array}{l}\text { Informations- } \\
\text { material }\end{array}$ & $\begin{array}{l}\text { Attraktiv gestaltete Broschüren } \\
\text { und Flyer, mit Informationen } \\
\text { zu psychoaktiven Substanzen, } \\
\text { Mischkonsum, Safer Use und } \\
\text { andere Risikofaktoren (z.B. Sa- } \\
\text { fer Sex). }\end{array}$ & $\begin{array}{l}\text { - Für die Zielgruppe } \\
\text { relevantes Wissen } \\
\text { schaffen } \\
\text { - Mythen abbauen } \\
\text { - Einen risikoarmen } \\
\text { Umgang fördern }\end{array}$ & $\begin{array}{l}\text { - Drugs Just Say } \\
\text { Know } \\
\text { - Informiere dich } \\
\text { bevor du konsu- } \\
\text { mierst } \\
\text { - Factsheets von } \\
\text { Suchtschweiz }\end{array}$ \\
\hline $\begin{array}{l}\text { Webseiten } \\
\text { Apps etc. }\end{array}$ & $\begin{array}{l}\text { Webseiten, Social Media Pro- } \\
\text { file, Apps, mit Informationen } \\
\text { zu psychoaktiven Substanzen, } \\
\text { Mischkonsum, Safer Use und } \\
\text { andere Risikofaktoren (z.B. Sa- } \\
\text { fer Sex). Z.T. mit Reflektions- } \\
\text { hilfen, wie Konsumtagebü- } \\
\text { cher, Kombi-Checker, On- } \\
\text { lineberatung }\end{array}$ & $\begin{array}{l}\text { - Informationen unab- } \\
\text { hängig von Ort und } \\
\text { Tageszeit zugängli- } \\
\text { che machen } \\
\text { - Konsumreflektion } \\
\text { mittels Online-Tools } \\
\text { fördern } \\
\text { - Niederschwellige, } \\
\text { da anonyme Bera- } \\
\text { tungsmöglichkeit }\end{array}$ & $\begin{array}{l}\text { - Suchtpräventions- } \\
\text { stellen des Kanton } \\
\text { Zürich } \\
\text { - Safezone.ch } \\
\text { - Saferparty.ch } \\
\text { - Eve\&Rave.ch } \\
\text { - Kombi-checker.ch } \\
\text { - Rave-it-safe.ch } \\
\text { - Know-drugs.ch }\end{array}$ \\
\hline Peer to Peer & $\begin{array}{l}\text { Einbezug von PartygängerIn- } \\
\text { nen, als BeraterInnen, in } \\
\text { Nightlife-Präventionsange- } \\
\text { bote. Oder Aufbau eines Netz- } \\
\text { werkes von Freunden als ei- } \\
\text { genständiges Nightlife-Prä- } \\
\text { ventionsangebot. }\end{array}$ & $\begin{array}{l}\text { - Sozialraumnähe } \\
\text { - Beratung auf } \mathrm{Au}- \\
\text { genhöhe } \\
\text { - Mediatoren }\end{array}$ & $\begin{array}{l}\text { - Eve \& Rave } \\
\text { Schweiz } \\
\text { - Aware Dance Cul- } \\
\text { ture } \\
\text { - Be my Angel } \\
\text { - Safer Dance Swiss }\end{array}$ \\
\hline Infostand & $\begin{array}{l}\text { Infostand, mit Beratungsmög- } \\
\text { lichkeit, Informationsmaterial, } \\
\text { aktuellen Warnungen, Kon- } \\
\text { dome und Ohrenstöpsel direkt } \\
\text { vor Ort. Teilweise mit einer } \\
\text { Ruhezone (Chill-Out) oder } \\
\text { Drug Checking Angebot. Ein- } \\
\text { zelne Projekte sind speziali- } \\
\text { siert auf den Konsum von Al- } \\
\text { kohol und die Sicherheit im } \\
\text { Strassenverkehr. }\end{array}$ & $\begin{array}{l}\text { - Verbesserung des } \\
\text { Zuganges zur Ziel- } \\
\text { gruppe mittels Prä- } \\
\text { senzen in deren Le- } \\
\text { benswelt } \\
\text { - Selektives Vermit- } \\
\text { teln von Informatio- } \\
\text { nen } \\
\text { - Früherkennung mit- } \\
\text { tels Kurzberatung } \\
\text { und Risikoassese- } \\
\text { ment. }\end{array}$ & $\begin{array}{l}\text { - Suchthilfe Region } \\
\text { Basel, Safer Dance } \\
\text { Basel } \\
\text { - Contact, Stiftung } \\
\text { für Suchthilfe, } \\
\text { Rave it Safe, Bern } \\
\text { - Stadt Zürich, Ju- } \\
\text { gendberatung } \\
\text { Streetwork, Safer- } \\
\text { party.ch } \\
\text { - Stiftung Alkohol } \\
\text { am Steuer nie, Be } \\
\text { my Angel, } \\
\text { Deutschschweiz }\end{array}$ \\
\hline
\end{tabular}




\begin{tabular}{|c|c|c|c|}
\hline Angebot & Beschreibung & Ziele & Referenzen \\
\hline $\begin{array}{l}\text { Psychedeli- } \\
\text { sche Ambu- } \\
\text { lanz }\end{array}$ & $\begin{array}{l}\text { Ergänzung eines Infostands an } \\
\text { mehrtägigen Festivals. Psycho- } \\
\text { soziale Betreuung von «über- } \\
\text { dosierten» Gästen, ohne } \\
\text { gleichzeitig vorliegendes me- } \\
\text { dizinisches Problem. Enge Zu- } \\
\text { sammenarbeit mit medizini- } \\
\text { schen Fachpersonal. Geschütz- } \\
\text { ter Chill-Out als Interventions- } \\
\text { und Rückzugsort. }\end{array}$ & $\begin{array}{l}\text { - Betreuung überdo- } \\
\text { sierter Gäste an ei- } \\
\text { nem Festival } \\
\text { - Unnötige Spitalein- } \\
\text { weisungen verhin- } \\
\text { dern } \\
\text { - Interdisziplinäre } \\
\text { Zusammenarbeit } \\
\text { vor Ort fördern }\end{array}$ & - Safer Dance Swiss \\
\hline $\begin{array}{l}\text { Aufsuchende } \\
\text { Sozial Arbeit } \\
\text { im Sozial- } \\
\text { raum Nacht- } \\
\text { leben }\end{array}$ & $\begin{array}{l}\text { Aufsuchende Soziale Arbeit an } \\
\text { den Orten des Nachtlebens, } \\
\text { z.B. im Club oder in deren } \\
\text { Umfeld, in den Nightlife- } \\
\text { Areas. Vermitteln von Infor- } \\
\text { mationen, Abgabe von Kondo- } \\
\text { men, evtl. Safer Use Materia- } \\
\text { lien. Teilweise auch ordnungs- } \\
\text { politische Teilaufgaben. }\end{array}$ & $\begin{array}{l}\text { - Verbesserung des } \\
\text { Zuganges } \\
\text { - Soziale Kontrolle im } \\
\text { öffentlichen Raum } \\
\end{array}$ & $\begin{array}{l}\text { - Safer Dance Basel } \\
\text { - Rave it Safe \& Pi- } \\
\text { not, Bern } \\
\text { - Jugendberatung } \\
\text { Streetwork \& SIP } \\
\text { Züri, Zürich } \\
\text { - SIP Luzern }\end{array}$ \\
\hline $\begin{array}{l}\text { Spezifische } \\
\text { Kampagnen, } \\
\text { z.B. Ist Luisa } \\
\text { hier? }\end{array}$ & $\begin{array}{l}\text { Spezifische Kampagnen wid- } \\
\text { men sich aktuellen Themen. Ist } \\
\text { Luisa hier? ist ein Hilfsangebot } \\
\text { welches sich primär an Frauen } \\
\text { in der Partyszene richtet. } \\
\text { Wenn eine sich bedrängt } \\
\text { fühlte Person nach Luisa fragt, } \\
\text { bietet das geschulte Personal } \\
\text { ihr einen Rückzugsort. An die- } \\
\text { sem Rückzugsort wird dann } \\
\text { das weitere Vorgehen bespro- } \\
\text { chen ohne das der Vorfall im } \\
\text { Mittelpunkt steht.15 }\end{array}$ & $\begin{array}{l}\text { - Niederschwellige } \\
\text { Unterstützung bei } \\
\text { sexueller Bedrän- } \\
\text { gung } \\
\text { - Sexualisierte Gewalt } \\
\text { in der Nacht thema- } \\
\text { tisieren }\end{array}$ & $\begin{array}{l}\text { - Bar \& Club Kom- } \\
\text { mission \& Frauen- } \\
\text { beratung Zürich } \\
\text { - Bar und Club Ver- } \\
\text { einigung \& Frau- } \\
\text { enzentrale Win- } \\
\text { terthur } \\
\text { - Safer Clubbing } \\
\text { Luzern \& Frauen- } \\
\text { zentrale Luzern }\end{array}$ \\
\hline $\begin{array}{l}\text { Drogeninfor- } \\
\text { mationszen- } \\
\text { tren }\end{array}$ & $\begin{array}{l}\text { Adaption des Informations- } \\
\text { stands, mit Drug Checking, in } \\
\text { ein Sozialraum unabhängiges } \\
\text { reguläres Setting. Informati- } \\
\text { onszentrum. Bieten zudem } \\
\text { weiterführende Beratungs- } \\
\text { möglichkeiten und meist auch }\end{array}$ & $\begin{array}{l}\text { - Erweiterung der } \\
\text { Zielgruppe } \\
\text { - Zugang unabhängig } \\
\text { von Anlass und } \\
\text { Szene } \\
\text { - Verbesserte Bera- } \\
\text { tungsmöglichkeiten }\end{array}$ & $\begin{array}{l}\text { - Jugendberatung } \\
\text { Streetwork, DIZ, } \\
\text { Zürich } \\
\text { - Contact Stiftung } \\
\text { für Suchthilfe, } \\
\text { DIB+, Bern } \\
\text { - Stiftung Suchhilfe } \\
\text { Region Basel, }\end{array}$ \\
\hline
\end{tabular}

${ }^{15}$ Ist Luisa da wurde von der Frauenberatungsstelle Münster ins Leben gerufen 


\begin{tabular}{|l|l|l|c|}
\hline Angebot & Beschreibung & Ziele & Referenzen \\
\hline & $\begin{array}{l}\text { themenspezifischen Sprech- } \\
\text { stunden. Oft integriert in ein } \\
\text { bestehendes, inhaltlich ergän- } \\
\text { zendes Angebot. }\end{array}$ & $\begin{array}{l}\bullet \text { Schnittstellen schaf- } \\
\text { fen, um eine Triage } \\
\text { zu erleichtern }\end{array}$ & $\begin{array}{l}\text { DIBS, Drogen- } \\
\text { Info-Basel-Stadt }\end{array}$ \\
\hline
\end{tabular}

Tabelle 1: Nightlife-Präventionsangebote in der Schweiz (Stand Februar 2020)

\subsubsection{Strukturelle Prävention im Schweizer Nachtleben}

Typische Beispiele für strukturelle Prävention im Nachtleben sind die Sensibilisierung - mittels Schulungen - von Bar- und Clubmitarbeitenden zum Umgang mit berauschten Gästen oder der Zugang zu kostenlosem Trinkwasser, Kondomen und Informationsmaterialien. Die Herausforderung dabei ist, Nachtkulturunternehmen vom Sinn solcher Massnahmen zu überzeugen. Zur Rolle der Sozialen Arbeit im Nachtleben gehören deshalb nicht nur die Pflege und die inhaltliche Weiterentwicklung struktureller Präventionsansätze, sondern auch die Vernetzung und Öffentlichkeitsarbeit. Denn es ist im Interesse der Nachtkulturunternehmen, dass eine breite Öffentlichkeit Kenntnis von Safer-Clubbing-Bestrebungen nimmt, da sich dies positiv auf das Image der Clubs und Bars auswirkt.

\begin{tabular}{|c|c|c|c|}
\hline Angebot & Beschreibung & Ziele & Referenzen \\
\hline $\begin{array}{l}\text { Round Table } \\
\text { Nightlife }\end{array}$ & \begin{tabular}{|l} 
Regelmässig stattfindende \\
Austauschtreffen. Im Fokus \\
steht meist das aktuelle Risiko- \\
verhalten der lokalen Par- \\
tygästen und die damit ver- \\
bundenen Probleme. Der \\
strukturelle Austausch wird \\
aber auch dazu genutzt andere \\
Themen zu diskutieren, wie \\
beispielsweise der Ausgangs- \\
lärm. ${ }^{16}$
\end{tabular} & $\begin{array}{l}\text { - Informationen di- } \\
\text { rekt aus dem Sozial- } \\
\text { raum erschliessen } \\
\text { - Regelmässiger Aus- } \\
\text { tausch zwischen Sta- } \\
\text { keholdern fördern } \\
\text { und strukturell ver- } \\
\text { ankern }\end{array}$ & $\begin{array}{l}\text { - Luzern, Safer } \\
\text { Clubbing Sektion } \\
\text { Luzern } \\
\text { - Winterthur, Bar } \\
\text { und Club Vereini- } \\
\text { gung (BCvW) } \\
\text { - Zürich, Jugendbe- } \\
\text { ratung Streetwork } \\
\text { \& Bar \& Club } \\
\text { Kommission. }\end{array}$ \\
\hline $\begin{array}{l}\text { Club Gütes- } \\
\text { igel, Festival } \\
\text { Labels }\end{array}$ & $\begin{array}{l}\text { Aktive Gestaltung von Club, } \\
\text { Bars und Events unter Einbe- }\end{array}$ & $\begin{array}{l}\text { - Minimieren von } \\
\text { umfeldbezogenen } \\
\text { Risiken }\end{array}$ & $\begin{array}{l}\text { - Safer Clubbing } \\
\text { Schweiz }\end{array}$ \\
\hline
\end{tabular}

\footnotetext{
${ }^{16}$ Round Table Nightlife existieren in der Schweiz in Luzern, in Zusammenhang mit der Safer Clubbing Sektion Luzern, in Winterthur, koordiniert durch die Bar und Club Vereinigung $(\mathrm{BcVW})$ und in Zürich organsiert durch die Jugendberatung Streetwork, Saferparty.ch und der Bar \& Club Kommission.
} 


\begin{tabular}{|c|c|c|c|}
\hline Angebot & Beschreibung & Ziele & Referenzen \\
\hline & $\begin{array}{l}\text { zug potentieller umfeldbezo- } \\
\text { gene Risken. Typische Bei- } \\
\text { spiele sind das Bereitstellen } \\
\text { von Informationen und Safer- } \\
\text { Use-Material, Personalschu- } \\
\text { lung und ein Zugang für } \\
\text { Nightlife-Präventionsange- } \\
\text { bote. }{ }^{17} \text { Im Vordergrund von } \\
\text { Labels im Festivalbereich }{ }^{18} \\
\text { liegt vor allem der Konsum } \\
\text { von Alkohol, die Jugend- } \\
\text { schutzrichtlinien und die Si- } \\
\text { cherheit im Strassenverkehr. }\end{array}$ & $\begin{array}{l}\text { - Selbstverantwor- } \\
\text { tung der Veranstal- } \\
\text { ter fördern } \\
\text { - Optimiertes Han- } \\
\text { deln des Personals } \\
\text { in Notsituationen } \\
\text { - Schaffen eines insti- } \\
\text { tutionsunabhängi- } \\
\text { gen Zugangs zur } \\
\text { Zielgruppe }\end{array}$ & $\begin{array}{l}\text { - Fiesta, Kanton } \\
\text { Wallis } \\
\text { - We can Dance it, } \\
\text { Genf }\end{array}$ \\
\hline $\begin{array}{l}\text { Bewusstes } \\
\text { Feiern }\end{array}$ & $\begin{array}{l}\text { Alternativen zur konsumorien- } \\
\text { tierten Feierkultur aufzuzei- } \\
\text { gen. Der Fokus liegt auf einem } \\
\text { besonders angenehmen Rah- } \\
\text { men, genügend Sitzplätzen, ei- } \\
\text { nem Chill Out, billigen oder } \\
\text { kostenlosen alkoholfreien Ge- } \\
\text { tränken, der Verzicht von Al- } \\
\text { kohol und der Möglichkeit der } \\
\text { Mitgestaltung. }\end{array}$ & $\begin{array}{l}\text { - Schaffen von Alter- } \\
\text { nativen zu einer } \\
\text { konsumorientierten } \\
\text { Feierkultur } \\
\text { - Bewusstes Feiern, } \\
\text { nicht nur auf der } \\
\text { Ebene des Konsums } \\
\text { psychoaktiver Sub- } \\
\text { stanzen }\end{array}$ & $\begin{array}{l}\text { - Aware Dance Cul- } \\
\text { ture, Bern } \\
\text { - Support your } \\
\text { Party, Zürich }\end{array}$ \\
\hline $\begin{array}{l}\text { Jugendbewil- } \\
\text { ligungen }\end{array}$ & $\begin{array}{l}\text { Jugendliche und junge Er- } \\
\text { wachsene, im Alter zwischen } \\
18 \text { und } 25 \text { Jahren, erhalten die } \\
\text { Möglichkeit legal Partys im öf- } \\
\text { fentlichen Raum durchzufüh- } \\
\text { ren. Je nach Stadt steht eine ge- } \\
\text { wisse Anzahl Daten und defi- } \\
\text { nierter Plätze zur Verfügung. } \\
\text { Dabei müssen die jugendli- } \\
\text { chen Veranstalter/innen ver- } \\
\text { schiedene Auflagen befolgen. }\end{array}$ & $\begin{array}{l}\text { - Möglichkeit der ak- } \\
\text { tiven Mitgestaltung } \\
\text { der Nacht durch Ju- } \\
\text { gendliche } \\
\text { - Nicht kommerzielle } \\
\text { Veranstaltungen } \\
\text { - Nutzen von Freiräu- } \\
\text { men }\end{array}$ & $\begin{array}{l}\text { - Stadt Zürich } \\
\text { - Stadt Bern }\end{array}$ \\
\hline
\end{tabular}

Tabelle 2: Strukturelle Prävention im Schweizer Nachtleben

\footnotetext{
17 Weitere Informationen www.safer-clubbing.ch

${ }^{18}$ Dazu zählen auch Dorffeste, Chilbi und die Fasnacht
} 


\subsection{Sozialräumliche Ansätze im Schweizer Nachtleben}

Die Soziale Arbeit nimmt eine wichtige Rolle ein, wenn es um die Anerkennung des Nachtlebens als Sozialraum und dessen nachhaltige Entwicklung geht. Da gerade heute in den immer dichter bewohnten Innenstädten Konflikte in Bezug auf die Nachtruhe praktisch zum Alltag gehören, steht die Koexistenz zwischen der Nachtkultur und der Nachbarschaft im Vordergrund einer sozialräumlichen Entwicklung. Dabei werden typische Methoden der Gemeinwesenarbeit eingesetzt. Beispiele sind Begehungen vor Ort, Mediation zwischen Konfliktparteien, das Zusammenbringen der Stakeholder, der strukturelle Austausch und die Unterstützung des Aufbaues von Bottom-Up-Strukturen wie beispielsweise Bar- und Clubkommissionen und Nachtstadträte als sich ergänzende Interessensvertretungen der Nacht.

\begin{tabular}{|c|c|c|c|}
\hline Angebot & Inhalt & Ziele & Referenzen \\
\hline $\begin{array}{l}\text { Bar und Club } \\
\text { Kommission }\end{array}$ & $\begin{array}{l}\text { Netzwerke von in der Nacht } \\
\text { tätigen Kulturunternehmen o- } \\
\text { der Festivals. Neben der Lob- } \\
\text { byarbeit, bieten sie auch Bera- } \\
\text { tungen für Mitglieder und spe- } \\
\text { zifische Weiterbildungen an. } \\
\text { Öffentlichkeitsarbeit, z.B. Tag } \\
\text { der offenen Bar und Club } \\
\text { Tür }{ }^{19} \text {. Einzelne Netzwerke } \\
\text { sind Kollektivmitglied bei Sa- } \\
\text { fer Clubbing Schweiz und set- } \\
\text { zen dies mittels Personalwei- } \\
\text { terbildungen und einer engen } \\
\text { Zusammenarbeit mit den loka- } \\
\text { len Nightlife-Präventionsange- } \\
\text { boten um. }\end{array}$ & $\begin{array}{l}\text { - Interessenvertretung } \\
\text { der Nachtkulturun- } \\
\text { ternehmen } \\
\text { - Image des Nachtle- } \\
\text { bens verbessern } \\
\text { - Ressourcenoptimie- } \\
\text { rung durch Zusam- } \\
\text { menarbeit }\end{array}$ & $\begin{array}{l}\text { - BCK Zürich } \\
\text { - BcVW Winterthur } \\
\text { - Nachtgallen } \\
\text { - BuCK Bern } \\
\text { - Kultur und Gast- } \\
\text { ronomie Basel } \\
\text { - Grand Conseil de } \\
\text { la Nuit Genf }\end{array}$ \\
\hline Nachtstadtrat & $\begin{array}{l}\text { Entweder eine einzelne Per- } \\
\text { son, auch bekannt als Night- } \\
\text { Major, oder ein Rat. Meistens }\end{array}$ & $\begin{array}{l}\text { - Interessenvertretung } \\
\text { des Nachtlebens } \\
\text { - Schnittstelle zwi- } \\
\text { schen den einzelnen }\end{array}$ & $\begin{array}{l}\text { - NachtStadtrat Zü- } \\
\text { rich }\end{array}$ \\
\hline
\end{tabular}

${ }^{19}$ Der Tag der offenen Bar und Club Tür wurde erstmals 2012 in Zürich, von der Bar \& Club Kommission durchgeführt. Seit 2018 in Kooperation mit dem EU-Netzwerk Live DMA als Open Club Day Gesamteuropäisch ausgerichtet Weitre Infos unter www.openclubday.ch 


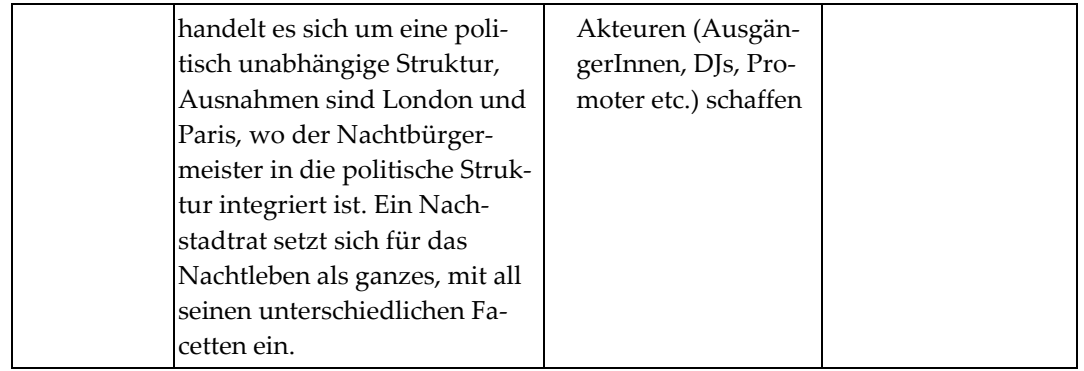

Tabelle 3: Sozialräumliche Ansätze im Schweizer Nachtleben

\subsection{Finanzierung der Sozialen Arbeit im Nachtleben}

Spenden der PartygängerInnen stellten in den 1990er-Jahren meist die einzige Finanzierungsmöglichkeit für die damaligen Szenenorganisationen dar. Teilweise beteiligten sich die Veranstalter an den Unkosten. Mit der Integration von Nightlife-Präventionsangeboten beispielsweise in die offene städtische Jugendarbeit wurden die anfallenden Kosten durch einen regulären Etat gedeckt oder die Angebote wurden durch kantonale oder städtische Behörden im Rahmen von Leistungsverträgen unterstützt. Für den Aufbau von Projekten spielte der damalige Impuls- und Entwicklungsfonds im Suchtbereich des Bundesamtes für Gesundheit eine wichtige Rolle. Viele der heutigen Nightlife-Präventionsangebote wurden zu Beginn über den Impulsfonds mitfinanziert. ${ }^{20}$ Heute verfügen die meisten Angebote der Sozialen Arbeit im Nachtleben über eine finanzielle Regeloder zumindest mehrjährige Projektversorgung. Doch ohne Freiwilligenarbeit geht es, zumindest bei den Festivalpräsenzen, auch heute nicht.

\section{Herausforderungen}

Erfreulich ist, dass in den letzten Jahren auch in der Schweiz neue Angebote der Sozialen Arbeit im Nachtleben ins Leben gerufen worden sind.

\footnotetext{
${ }^{20}$ Impulsfonds «Therapie und Schadensminderung» im Auftrag des BAG: für den Aufbau neuartiger Angebote, zur Erweiterung bzw. Differenzierung bestehender Leistungsangebote, für die interkantonale Vernetzung von Angeboten und zur Erarbeitung praxisbezogener Instrumente und Grundlagen (2005 - 2016).
} 
Begrüssenswert ist auch, dass der Fokus dabei nicht mehr nur auf den Problemen liegt, sondern das Nachtleben zunehmend als wichtiger Bestandteil unserer Gesellschaft anerkannt wird, welches eine bedeutende Rolle spielt, wenn es um die Zufriedenheit der urbanen Bevölkerung geht. 2015 waren in der Schweiz 18 Institutionen präventiv oder schadensmindernd im Nachtleben aktiv. Daraus resultierten rund 132825 Kontakte und 7142 Kurzberatungen ${ }^{21}$ (vgl. Infodrog 2016:9). Im Rahmen von Drug Checkings wurden 20172242 Analysen durchgeführt, wobei 1379 Warnungen aufgrund sehr hoher Dosierungen oder unerwarteter Wirkstoffen ausgesprochen wurden (vgl. Infodrog 2017:25).

\subsection{Erreicht die Soziale Arbeit ihre Zielgruppe?}

Ja. Die in der Schweiz zur Verfügung stehenden Daten zum Drogenkonsum belegen, dass es sich bei den NutzerInnen von Nightlife-Präventionsangeboten - im Vergleich zur Schweizer Bevölkerung insgesamt - um eine drogenaffine Bevölkerungsgruppe handelt. Die Praxis zeigt, dass der Kontakt vor Ort für die meisten PartygängerInnen die erste Inanspruchnahme des professionellen Hilfesystems darstellt. Diese zuvor nur schwer zu fassende Risikogruppe kann dank Nightlife-Präventionsangeboten erreicht werden, was Prävention und Schadensminderung in dieser Zielgruppe erst möglich macht. Die dabei mit dem standardisierten Fragebogen erhobenen Daten sind zudem eine wichtige Ergänzung zum nationalen Monitoring, wie beispielsweise ein Vergleich mit dem Schweizer Suchtmonitoring $^{22}$ aus dem Jahr 2013 zeigt (vgl. Maier et al 2014: 13).

\subsection{Wirksamkeit von Nightlife-Präventionsangeboten}

Eine Aussage zur Wirksamkeit von Nightlife-Präventionsangeboten ist nur sehr eingeschränkt möglich. Der Hauptgrund dafür liegt in der schwierigen Messbarkeit von Verhaltensveränderungen innerhalb der Zielgruppe. Da wie im Kapitel 3.1 aufgeführt nur bei einem kleinen Teil

\footnotetext{
${ }^{21}$ Von einer Beratung wird gesprochen, wenn ein Informationsgespräch länger als $15 \mathrm{Minu-}$ ten dauert.

222017 wurde das Monitoring vom MonAM - Monitoring-System Sucht und NCD abgelöst.
} 
der Freizeitdrogenkonsumierenden tatsächlich eine Veränderung des persönlichen Risikoverhaltens angezeigt ist, geht es primär darum, zu einer nur schwierig erfassbaren Reflexion des Konsums beizutragen. Besser messbar ist, wie häufig im Rahmen einer Substanzanalyse vor einer besonders gefährlichen Substanz gewarnt werden muss. ${ }^{23}$ Einen Hinweis auf die Effektivität von Nightlife-Präventionsangeboten gibt eine interne Evaluation der Stadt Zürich aus dem Jahr 2010, welche erfreulicherweise feststellen konnte, dass der besonders riskante Mischkonsum um rund 20 Prozent abgenommen hatte. Eine wichtige Rolle schienen dabei das stationäre und mobile Drug Checking zu spielen, da die Analyseresultate verbunden mit erlebten Wirkungen die Reflexion des persönlichen Konsums besonders stimuliert. Die Attraktivität der Substanzanalyse erleichtert den Partydrogenkonsumierenden auch den Kontakt, dies spiegelt sich in der seit 2001 stetig steigenden Nachfrage wieder (Bücheli et al. 2010).

\subsection{Strukturelle Lücken}

Trotz der Wichtigkeit von Substanzanalysen ist ein Drug Checking nur in der Stadt Zürich, in den Kantonen Basel-Stadt und Basel Landschaft, im Kanton Bern und im Kanton Genf möglich. Neben einer verbesserten nationalen Abdeckung von Drug-Checking-Angeboten besteht auf nationaler Ebene Handlungsbedarf in Bezug auf ein nationales Frühwarnsystem für illegale Substanzen. Substanzwarnungen werden bisher der Fachwelt mittels institutionseigener Mailverteiler, beispielsweise von der Jugendberatung Streetwork, zur Verfügung gestellt. Es fehlt eine nationale Stelle, die Warnungen strukturiert, interpretiert und dreisprachig zur Verfügung stellt. Zudem gibt es Lücken im Bereich von gender- und migrationsgerechten Angeboten. Gewalt im Nachtleben ist ein wichtiges Thema und wird im Rahmen der aufsuchenden ordnungspolitischen nächtlichen Sozialen Arbeit ${ }^{24}$, im Rahmen des Management des nächtlichen öffentlichen Raums und innerhalb von Beratungen der Nightlife-Präventionsangeboten durchaus thematisiert, es gibt aber kein spezifisches Angebot dazu.

\footnotetext{
${ }^{23} 2018$ warnte die Jugendberatung Streetwork, saferparty.ch bei 1004 von insgesamt 2199 analysierten Substanzen. Die meisten Warnungen entfielen dabei auf hochdosierte XTC-Tabletten und Kokainproben mit dem Streckmittel Levamisol.

${ }^{24}$ Hiermit sind Angebote wie die SIP Züri, SIP Luzern oder Pinto in Bern gemeint.
} 
Aufgrund der professionellen Strukturen der meisten Präventions- und Schadensminderungsangebote gibt es Lücken in der Vor-Ort-Präsenz an illegalen, meist sehr kurzfristig angesetzten Underground-Anlässen. Entweder haben die Fachleute von diesen Anlässen keine Kenntnisse, ihr Auftrag bezieht sich auf legale Angebote und/oder die Reaktionszeit ist zu langsam. Alkohol ist und bleibt die Partydroge Nummer 1. Neben BonusMalus-Ansätzen ${ }^{25}$, die z. B. einen moderaten Alkoholkonsum mit Punkten belohnen, und Angeboten zum Thema Alkohol am Steuer ${ }^{26}$ fehlt es an überzeugenden individualisierten schadensmindernden Ansätzen. Dies gilt auch für den Tabakkonsum. Obwohl dieser im Nachtleben weit verbreitet ist, wird er nur selten thematisiert. Dies hängt auch damit zusammen, dass von der Fachwelt akzeptierte schadensmindernde Ansätze im Tabakbereich fehlen.

\section{Ausblick}

Die über 20-jährige Geschichte der Schadensminderung im Schweizer Nachtleben ist geprägt durch eine Fokussierung auf das individuelle Risikoverhalten. Aktuell steht in der öffentlichen Diskussion die Belastung des öffentlichen Raumes in der Nacht im Zentrum. Dabei geht es primär um Lärm, aber auch Gewalt und Alkoholexzesse sind Thema. Die in den letzten Jahren aufgrund dieser Diskussion vorgestellten Nachtleben-Konzepte ${ }^{27}$ befassen sich oft einseitig mit Regulierungen, wie z. B. der (Wieder)Einführung einer Sperrstunde. Da die verlängerten Öffnungszeiten, welche in den 1980er-Jahren hart erkämpft worden waren, immer noch einem gesellschaftlichen Bedürfnis entsprechen und zu einer besseren Verteilung von Personenströmen in der Nacht führen, stellt sich die Frage, wie nachhaltig solch einseitig formulierte Konzepte sind. Eine zunehmende Regulierung würde zu Ausweichbewegungen, zum entstehen illegaler Szenen führen, die wiederum unvorhersehbare neue Probleme mit sich ziehen. Um dies zu verhindern, sollten im Interesse der Sozialen Arbeit Lösungen

\footnotetext{
${ }^{25}$ Z. B. Poinzz.

${ }^{26}$ Z. B. «be my angel tonight».

${ }^{27}$ Z. B. das Nachtlebenkonzept der Stadt Bern oder des Schweizer Städteverbands.
} 
auf der Grundlage eines Multi-Stakeholder-Kooperations-Prozesses entwickelt werden. Dank ihrer breiten Akzeptanz im Nachtleben kann die Soziale Arbeit nicht nur bei der Initiierung solcher Prozesse eine wichtige Rolle spielen. In Bezug auf eine Verbesserung der nächtlichen Rahmenbedingungen sollte sich eine Soziale Arbeit nach Acht auch für eine kohärente Reglementierung psychoaktiver Substanzen einsetzen. Mittels eines kontrollierten Marktes für weitgehend alle Substanzen, liesse sich das Spannungsfeld legale versus illegale Substanzen lösen. Die Arbeit für die Soziale Arbeit würde sich dadurch vereinfachen, da die Realität eines illegalen Substanzkonsums von den Nachtkulturunternehmen nicht mehr verschwiegen und der Zugang für Nightlife-Präventionsangebote nicht mehr dauernd gerechtfertigt werden müsste. Für einen kontrollierten Markt spricht auch, dass sich Drug Checking kapazitätsmässig nie so flächendeckend ausweiten lässt, dass alle Schweizer Freizeitdrogenkonsumierenden Zugang zu einem solchen Angebot hätten.

\section{Literatur}

Bachmann, A./Bücheli, A. (2011): Frühintervention im Nachtleben. SuchtMagazin 37(5): 2730 .

Bücheli, A. (2009): Von der Nacht zum Tag. Welches gemeinwesenentwicklerische Potential steckt in der Zürcher Clubkultur? Hochschule München: Masterthesis.

Bücheli, A. (2014): Schadensminderung im Schweizer Nachtleben. SuchtMagazin 40(2): 1623.

Bücheli, A. (2015): Die gesunde Stadt und das Nachtleben. Stadt:pilot spezial. Die Stadt und das Nachtleben. Das Magazin zu den Pilotprojekten der Nationalen Stadtentwicklung. Sonderausgabe/September 2015: 25-26. www.bit.ly/2F31Y0k, Zugriff 07.04.2019.

Bücheli, A. (2017): Risikoarmer Drogengebrauch. Für eine genussoptimierte Haltung. Solothurn: Nachtschatten.

Bücheli, A./Hochenegger, M./Nunes, S. (2017): Nightlife-Prävention im deutschsprachigen Raum. SuchtMagazin 43(2\&3): 43-48.

Infodrog (2017): Safer Nightlife Schweiz. Factsheet Drug Checking. Bern: Infodrog. www.tinyurl.com/yyw34ruk, Zugriff 01.02.2020.2019.

Infodrog (2016): Tätigkeitsbericht Safer Nightlife Schweiz 2014/2015. Bern: Infodrog. www.bit.ly/2QrL3pG, Zugriff 07.04.2019.

Infodrog (2017): Tätigkeitsbericht Safer Nightlife Schweiz 2017. Bern: Infodrog. www.tinyurl.com/yx96vfj5, Zugriff 01.02.2020

Kosnick, K. (2012): Utopien des Sozialen. Das Nachtleben als Sozialisationsinstanz und Spiegel von Gesellschaft? Vortrag auf der Konferenz Safer Nightlife Schweiz, Biel, 19.September 2012. www.tinyurl.com/cpbnyk3, Zugriff 07.04.2019. 
Maier, L.J./Bachmann, A./Bücheli, A./Schaub, M.P. (2014): Erarbeitung Instrumente zur Früherkennung und Frühintervention von problematischem Substanzkonsum im Schweizer Nachtleben (2011-2013). Bern: Infodrog. www.doi.org/10.5167/uzh-102177

Maier, L.J./Bachmann, A./Lischer, A./Humm, M. (2017): Substanzkonsum in der Freizeit: SuchtMagazin 43(1): 20-24.

Müller, C. P./Schumann, G. (2011). Drugs as instruments: a new framework for non-addictive psychoactive drug use. The Behavioral and Brain Sciences, 34(6): 293-310. www.doi.org/10.1017/S0140525X11000057.

Schori, D. (2019): Drug Checking in der Schweiz: SuchtMagazin 45(6): 10-16.

Schweizerischer Städteverband (2012): Städtisches Nachtleben. Situationsanalyse und mögliche Vorgehensweisen. Schweizerischer Städteverband. www.tinyurl.com/cpv25gs, Zugriff 07.04.2019.

Wollschläger, M./Schori, D. (2019): Konsum von psychoaktiven Substanzen in der Freizeit: Auswertung der Befragungen von Konsumierenden 2018. Bericht 2019. 5-19. Bern: Infodrog., www.bit.ly/2ubO9Z3, Zugriff 05.02.2020.

Open Access Dieses Kapitel wird unter der Creative Commons Namensnennung 4.0 International Lizenz (http://creativecommons.org/licenses/by/4.0/deed.de) veröffentlicht, welche die Nutzung, Vervielfältigung, Bearbeitung, Verbreitung und Wiedergabe in jeglichem Medium und Format erlaubt, sofern Sie den/die ursprünglichen Autor(en) und die Quelle ordnungsgemäß nennen, einen Link zur Creative Commons Lizenz beifügen und angeben, ob Änderungen vorgenommen wurden.

Die in diesem Kapitel enthaltenen Bilder und sonstiges Drittmaterial unterliegen ebenfalls der genannten Creative Commons Lizenz, sofern sich aus der Abbildungslegende nichts anderes ergibt. Sofern das betreffende Material nicht unter der genannten Creative Commons Lizenz steht und die betreffende Handlung nicht nach gesetzlichen Vorschriften erlaubt ist, ist für die oben aufgeführten Weiterverwendungen des Materials die Einwilligung des jeweiligen Rechteinhabers einzuholen.

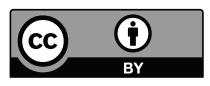

